\title{
Multi-relational data mining in Microsoft SQL Server 2005
}

\author{
C. L. Curotto ${ }^{1} \&$ N. F. F. Ebecken ${ }^{2} \&$ H. Blockeel ${ }^{3}$ \\ ${ }^{1}$ PPGCC/UFPR, Universidade Federal do Paraná, Brazil \\ ${ }^{2}$ COPPE/UFRJ, Universidade Federal do Rio de Janeiro, Brazil \\ ${ }^{3}$ DTAI/DCS/KUL, Katholieke Universiteit Leuven, Belgium
}

\begin{abstract}
Most real life data are relational by nature. Database mining integration is an essential goal to be achieved. Microsoft SQL Server (MSSQL) seems to provide an interesting and promising environment to develop aggregated multi-relational data mining algorithms by using nested tables and the plug-in algorithm approach. However, it is currently unclear how these nested tables can best be used by data mining algorithms. In this paper we look at how the Microsoft Decision Trees (MSDT) handles multi-relational data, and we compare it with the multi-relational decision tree learner TILDE. In the experiments we perform, MSDT has equally good predictive accuracy as TILDE, but the trees it gives either ignore the relational information, or use it in a way that yields non-interpretable trees. As such, one could say that its explanatory power is reduced, when compared to a multi-relational decision tree learner. We conclude that it may be worthwhile to integrate a multi-relational decision tree learner in MSSQL.
\end{abstract}

Keywords: multi-relational, data mining, algorithm, decision trees, databases, sql server, nested tables.

\section{Introduction}

To achieve the tight coupling of Data Mining (DM) techniques in Database Management Systems (DBMS) technology, a number of approaches have been developed in the last years. These approaches include solutions provided by both company and academic research groups.

Toward this objective, the Microsoft (MS) Object Linking and Embedding Database for DM (OLE DB DM) technology provides an industry standard for 
developing DM algorithms [1]. This technology was first included in the MSSQL 2000 release [2]. The MSSQL Analysis Services (SSAS) has included a DM provider supporting two DM algorithms (one for classification by decision trees and another for clustering) and the DM aggregator feature made possible for developers and researchers to implement new DM algorithms. The MSSQL 2005 version [2] has included more seven algorithms as well as a new way to aggregate new algorithms, using a plug-in approach instead of DM providers.

Subscription
\begin{tabular}{|l|l|l|}
\hline Number & Name & Course \\
\hline 1 & adams & erm \\
\hline 2 & adams & so2 \\
\hline 3 & adams & sw \\
\hline 4 & blake & sw \\
\hline 5 & blake & erm \\
\hline 6 & king & sw \\
\hline 7 & king & erm \\
\hline 8 & king & so2 \\
\hline 9 & smith & $c s 0$ \\
\hline 10 & miller & so2 \\
\hline 11 & scott & $c s 0$ \\
\hline 12 & scott & abc \\
\hline 13 & turner & cso \\
\hline 14 & turner & abc \\
\hline
\end{tabular}

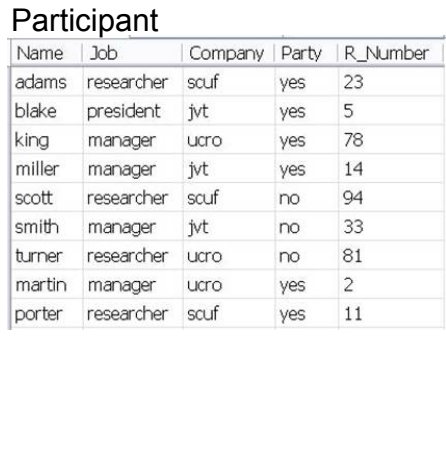

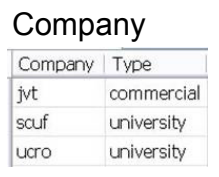

Course

\begin{tabular}{|l|l|l|}
\hline Course & Lenath & Type \\
\hline $\mathrm{abc}$ & 2 & advanced \\
\hline $\mathrm{cso}$ & 2 & introductory \\
\hline $\mathrm{erm}$ & 3 & introductory \\
$\mathrm{so} 2$ & 3 & introductory \\
\hline $\mathrm{srw}$ & 3 & advanced \\
\hline
\end{tabular}

Figure 1: Registration - Four tables relational data.

On the other hand, relational data impose some additional effort to deal with them. While conventional classifiers assume that data sets are recorded in single flat files or tables, a relational classifier has to face with more complex data structures as shown by the simple Registration relational dataset [3] represented by four tables in Fig. 1. In order to predict which participants are going to attend a party, one needs participant personal data provided by table Participant, participant employer data provided by table Company, participant course subscriptions data provided by table Subscription and course data provided by table Course.

The usual approach to solve this problem is to use a single flat table, as shown in Fig. 2, assembled by performing a relational join operation on the four tables. But this approach may produce extremely large and impractical to handle tables, with lots of repeated and null data.

In consequence of those problems, multi-relational DM (MRDM) approaches have been receiving considerable attention in the literature [3-11]. These approaches rely on developing specific algorithms to deal with the relational feature of the data.

By another way, OLE DB DM technology supports nested tables (also known as table columns). As shown in Fig. 3, the row sets represent uniquely the tables in a nested way. There are no redundant or null data for the participant in each row set. One row per participant is all that is needed, and the nested columns of the row set contain the data pertinent to that participant.

In the next items, it will be presented a brief overview of the approaches used to deal with MRDM, as well as the results of experiments carried out to 
evaluate the scope of OLE DB DM approach. In these experiments the results of MSDT algorithm are compared with those produced by TILDE [4]. Finally, the conclusion is shown together with some recommendations to make improvements in this field of research.

\begin{tabular}{|l|l|l|l|l|l|l|l|}
\hline Name & Job & Companv & Tvpe & Party & Course & Length & CourseTvpe \\
\hline adams & researcher & scuf & university & yes & erm & 3 & introductory \\
\hline adams & researcher & scuf & university & yes & so2 & 3 & introductory \\
\hline adams & researcher & scuf & university & yes & srw & 3 & advanced \\
\hline blake & president & jvt & commercial & yes & srw & 3 & advanced \\
\hline blake & president & jut & commercial & yes & erm & 3 & introductory \\
\hline king & manager & ucro & university & yes & srW & 3 & advanced \\
\hline king & manager & ucro & university & yes & erm & 3 & introductory \\
\hline king & manager & ucro & university & yes & so2 & 3 & introductory \\
\hline martin & manager & ucro & university & yes & NULL & NULL & NULL \\
\hline miller & manager & jvt & commercial & yes & so2 & 3 & introductory \\
\hline porter & researcher & scuf & university & yes & NULL & NULL & NULL \\
\hline scott & researcher & scuf & university & no & cso & 2 & introductory \\
\hline scott & researcher & scuf & university & no & abc & 2 & advanced \\
\hline smith & manager & jut & commercial & no & cso & 2 & introductory \\
\hline turner & researcher & ucro & university & no & cso & 2 & introductory \\
\hline turner & researcher & ucro & university & no & abc & 2 & advanced \\
\hline
\end{tabular}

Figure 2: Registration - Unique table.

\begin{tabular}{|c|c|c|c|c|c|c|c|}
\hline Name & Job & Company & Type & Party & Course & Length & CourseType \\
\hline \multirow{3}{*}{ adams } & \multirow{3}{*}{ researcher } & \multirow{3}{*}{ scuf } & \multirow{3}{*}{ university } & \multirow{3}{*}{ yes } & erm & 3 & introductory \\
\hline & & & & & so2 & 3 & introductory \\
\hline & & & & & srw & 3 & advanced \\
\hline \multirow{2}{*}{ blake } & \multirow{2}{*}{ president } & \multirow{2}{*}{ jvt } & \multirow{2}{*}{ commercial } & \multirow{2}{*}{ yes } & srw & 3 & advanced \\
\hline & & & & & erm & 3 & introductory \\
\hline \multirow{3}{*}{ king } & \multirow{3}{*}{ manager } & \multirow{3}{*}{ ucro } & \multirow{3}{*}{ university } & \multirow{3}{*}{ yes } & srw & 3 & advanced \\
\hline & & & & & erm & 3 & introductory \\
\hline & & & & & so2 & 3 & introductory \\
\hline martin & manager & ucro & university & yes & & & \\
\hline miller & manager & jvt & commercial & yes & so2 & 3 & introductory \\
\hline porter & researcher & scuf & university & yes & & & \\
\hline \multirow{2}{*}{ scott } & \multirow{2}{*}{ researcher } & \multirow{2}{*}{ scuf } & \multirow{2}{*}{ university } & \multirow{2}{*}{ no } & cso & 2 & introductory \\
\hline & & & & & $a b c$ & 2 & advanced \\
\hline smith & manager & jvt & commercial & no & cso & 2 & introductory \\
\hline \multirow{2}{*}{ turner } & \multirow{2}{*}{ researcher } & \multirow{2}{*}{ ucro } & \multirow{2}{*}{ university } & \multirow{2}{*}{ no } & cso & 2 & introductory \\
\hline & & & & & $a b c$ & 2 & advanced \\
\hline
\end{tabular}

Figure 3: Registration - Nested table.

\section{Multi-relational DM approaches}

\subsection{Unique table approach}

Most classifiers work on a single table (attribute-value learning) with a fixed set of attributes. So it is restrictive in DM applications with multiple tables. It is 
possible to construct, by hand, a single table by performing a relational join operation on multiple tables using propositional logic as shown in Fig. 2.

For one-to-one and many-to-one relationships, one can join in the extra fields to the original relation without problems. For one to many relationships, there are two ways to handle them. The first one is just compute the join, but this leads to data redundancy, missing values, statistical skew, and loss of meaning. A single instance in the original database is mapped onto multiple instances in the new table, which is problematic. The second way is aggregate the information in different tuples representing the same individual into one tuple after computing the join. This removes the problems mentioned above, but causes loss of information because details originally present have been summarized away.

\subsection{Multi-relational DM algorithms}

This is a group of several approaches [5]: Propositional Learning; Inductive Logic Programming (ILP); Multi-Relational DM (MRDM); First Order Bayesian Networks (FOBN).

The Propositional Learning approach is a two independent step process, in which the initial one produces automatically a flat table that can be processed in the second step by any DM algorithm. It is essentially the same as described in the unique table approach, and has the same problems.

The ILP [3,4,7] approaches handle multiple tables directly, using a first order logic language to describe patterns extending over multiple tuples. These approaches include: Progol, First Order Inductive Logic (FOIL), TILDE [4], Inductive Constraint Logic (ICL), and CrossMine [8].

The MRDM approaches include a number of variants such as those presented by the following studies: Multi-Relational Decision Tree Induction [9], Multi-Relational Decision Tree (MRDT) [5], Multi-Relational Naïve Bayes Classifier (Mr-SBC) [10], and Multi-Relational Model Trees with support to regression (Mr-SMOTI) [11]. They do essentially the same as the IPL approaches do, but work in the relational database setting.

Finally, FOBN approaches extend the ILP or MRDM approaches by combining them with probabilistic (Bayesian) reasoning. These approaches include: Probabilistic Relational Model (PRM) [12], Probabilistic Logic Program (PLP), Bayesian Logic Program (BLP), and Stochastic Logic Program (SLP).

\subsection{OLE DB DM nested tables}

MS OLE DB DM uses nested DM columns (nested tables). DM models (DMMs) use this nested column structure for both input and output data, as the syntax used to populate a DMM with training data allows nested columns to be represented as sub-queries. DM algorithms cannot work directly with this approach. Unnesting them using the traditional unnest operator yields a single table in the same format as the join approach mentioned above, with the same problems. To avoid these problems is used a sparse matricial approach. 
First of all are produced expanded versions of the main (case and nested) tables by joining them on their support tables. Support tables are those that the main tables hold one-to-one or many-to-one relationship with them. The lines of this matrix are the expanded case table records and the matrix columns are the compound attributes. These compound attributes are the expanded case table normal attributes plus additional attributes that correspond to all elements of the expanded nested tables mapped by columns.

To exemplify this approach the relational Registration DMM shown in Fig. 4 will be used. The Company support table is joined with Participant case table to produce its expanded version, addind more one attribute (CompanyType) to the original main table. The join of Subscription nested table with Course support table adds more two attributes (Lenght and Type) to the nested table producing its expanded version.

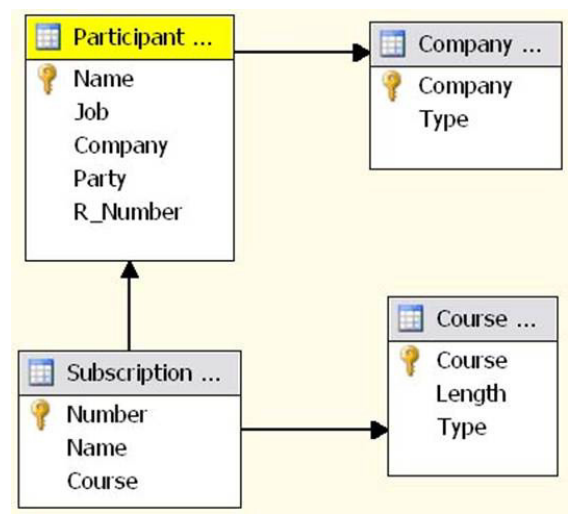

Figure 4: Registration - DMM schema. Participant is the case table, Subscription is the nested table and Course and Company are the support tables.

\begin{tabular}{|c|c|c|c|c|c|c|}
\hline \multicolumn{2}{|c|}{} & \multicolumn{5}{|c|}{ Attributte number/attribute name } \\
\cline { 4 - 7 } & 0 & 1 & 2 & 3 \\
\hline Num & Case & $\begin{array}{c}\text { Num of } \\
\text { attributes }\end{array}$ & Party & Company & Job & CompanyType \\
\hline 1 & 1:adams & 16 & 2:yes & 2:scuf & 3:researcher & 2: university \\
\hline 2 & 2:blake & 12 & 2:yes & 1:jvt & 2:president & 1:commercial \\
\hline 3 & 3:king & 16 & 2:yes & 3:ucro & 1:manager & 3: university (2:) \\
\hline 4 & martin & 4 & 2:yes & 3:ucro & 1:manager & 3: university (2:) \\
\hline 5 & 4:miller & 8 & 2:yes & 1:jvt & 1:manager & 1:commercial \\
\hline 6 & porter & 4 & 2:yes & 2:scuf & 3:researcher & 2: university \\
\hline 7 & $5:$ scott & 12 & 1:no & 2:scuf & 3:researcher & 2: university \\
\hline 8 & 6:smith & 8 & 1:no & 1:jvt & 1:manager & 1:commercial \\
\hline 9 & 7:turner & 12 & 1:no & 3:ucro & 3:researcher & 3: university (2:) \\
\hline
\end{tabular}

Figure 5: Registration - Sparse matrix normal attributes.

The Registration sparse matrix has nine lines (the dataset cases) and sixty attributes. Four of them are the normal attributes (Fig. 5) and fifty-six are the additional attributes mapped from the columns of the expanded nested table (Fig. 6). These fifty-six attributes correspond to the fourteen records times the four 
attributes of the Subscription expanded nested table. The name of each additional attribute is composed by the name of the nested table plus the name (or number) of its key attribute (ID) plus the name of the attribute considered as it can be seen in Fig. 6. Some problems with attribute values could be observed. In Fig. 5 the CompanyType attribute must have only two different values but three values were retrieved from the dataset. This same behaviour was observed in several others attributes as it can be seen in Fig. 6. The reason for this unexpected behaviour is not clear to us.
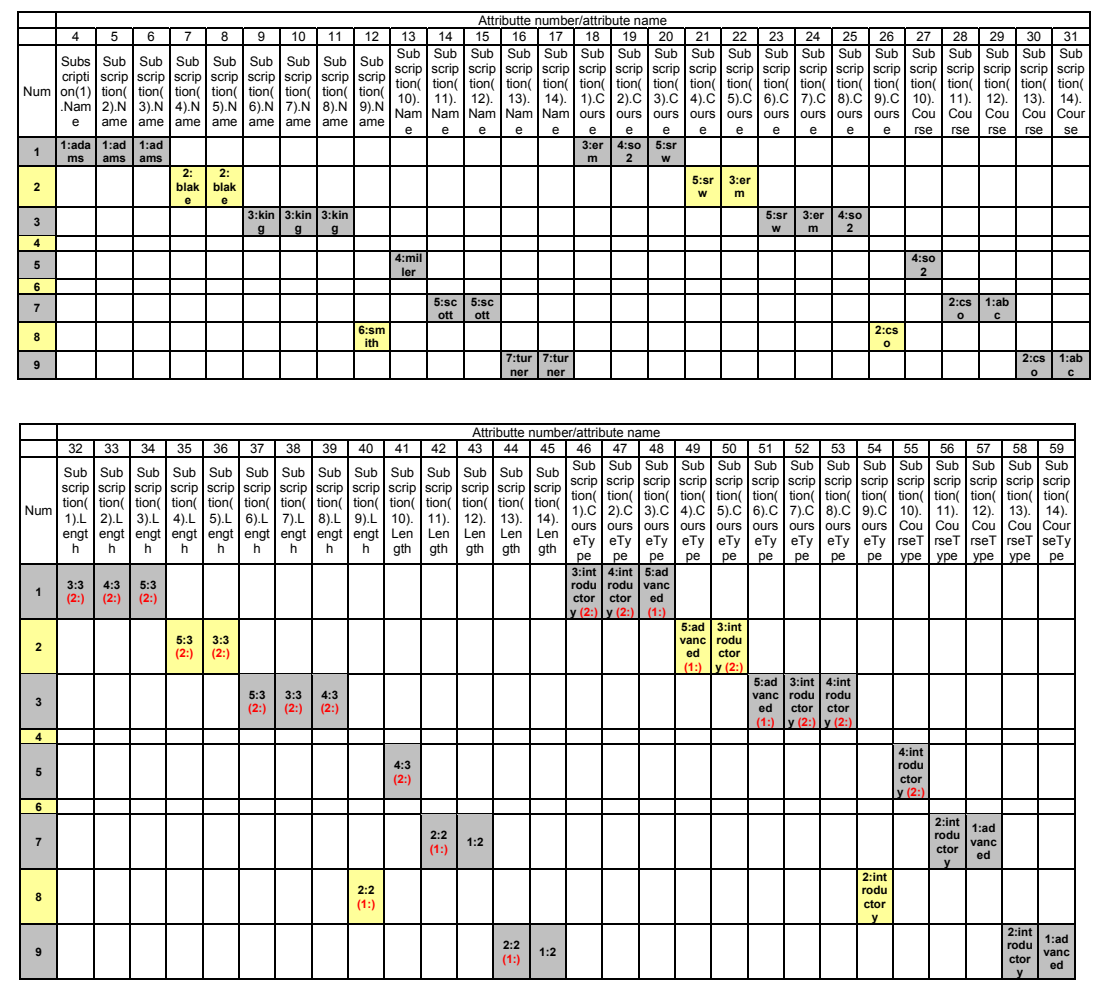

Figure 6: Registration - Sparse matrix additional attributes.

\section{Computational experiments}

Two experiments were carried out in order to evaluate the scope of MSSQL while dealing with MRDM. Two datasets are used: Registration [3] and Mutagenesis [13]. The numeric results and the decision trees obtained by MSDT (using the sparse matrix) in these experiments were compared with those produced by TILDE (using the original data). These experiments were made by using an IBM PC compatible microcomputer, Intel Pentium M $2.00 \mathrm{GHz}$ processor inside, 1.0 GB of RAM memory, 1.5 GB virtual memory, $100 \mathrm{MB}$ hard disk, MS Windows XP Pro SP2 and MSSQL 2005 Enterprise installed. 


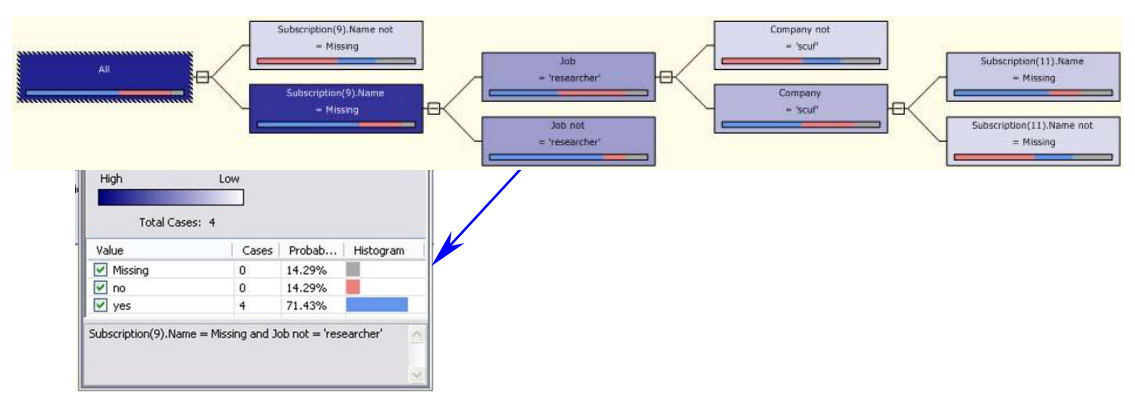

Figure 7: Registration-MSDT Decision tree.

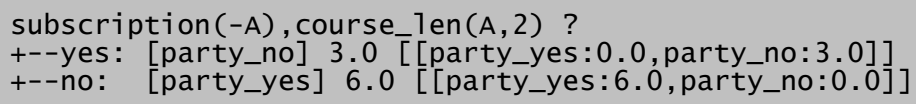

Figure 8: Registration - TILDE Decision tree.

\subsection{Registration dataset}

Both MSDT and TILDE achieved full accuracy while training this dataset and using itself as the test dataset. TILDE showed a meaningful decision tree (Fig. 8), stating that a participant attends the party if he or she has a subscription to a course of length 2, while MDST showed attributes in its decision tree (Fig. 7) that are difficult to interpret, e.g., subscription(9).Name not= missing has no real meaning because the subscription numbering is not defined globally.

\subsection{Mutagenesis dataset}

This dataset concerns with the problem of identifying the mutagenic compounds and have been extensively used to test both ILP and MRDM systems. It was considered, analogously to related experiments in the literature, the regression friendly dataset of 188 elements. It was used the background knowledge $\mathrm{BK}_{2}$, which consists of those data obtained with the modelling package Quanta, plus indicators ind1, indA and attributes logp and lumo [11]. Only two classes were considered for the prediction attribute: positive or negative. We used ten-fold cross-validation to estimate the accuracy of the classifiers. The DMM schema is shown in Fig. 9. Some parameters were the same for both MSDT1 and MSDT2: COMPLEXITY_PENALTY $=0.1$, MAXIMUM_INPUT_ATTRIBUTES $=255$, MAXIMUM_OUTTPUT_ATTRIBUTES $=255, \quad$ SCORE_METHOD $=4$ and SPLIT_METHOD $=3$. MSDT1 uses MINIMUM_SUPPORT $=10$ and MSDT2 uses MINIMUM_SUPPORT $=1$. MSDT1 configuration uses all input attributes and MSDT2 uses only Atom and Bond input attributes.

MSDT1 achieved $87 \pm 6 \%$ of accuracy while MSDT2 got $66 \pm 13 \%$ (because the Molecule input attributes were deliberately ignored). TILDE got $80 \pm 3 \%$ of 
accuracy. Figs. 10 to 12 show one of the ten-fold cross validation decision trees produced by the corresponding classifiers. TILDE showed a meaningful decision tree (Fig. 12) compared with those produced by MDST (Fig. 10 and 11). By example, tests such as atom(d104_38).Type not $=$ missing (Fig. 11) are not interpretable because there is no natural numbering for the atoms. This illustrates the loss of meaning: atom \#n has a meaning in the current representation of the data (it is the n'th atom in our ordered list), but not in the real world (because the atoms in a molecule are not actually ordered). Multi-relational trees, of which the TILDE trees shown in Fig. 12 are an example, avoid this problem: they refer to atoms not with some specific ID (e.g., d104_38) but through variables and properties of these (e.g., a C-atom of type 35) that always have meaning. That is the crucial difference between the MSDT trees shown here, and multi-relational trees.

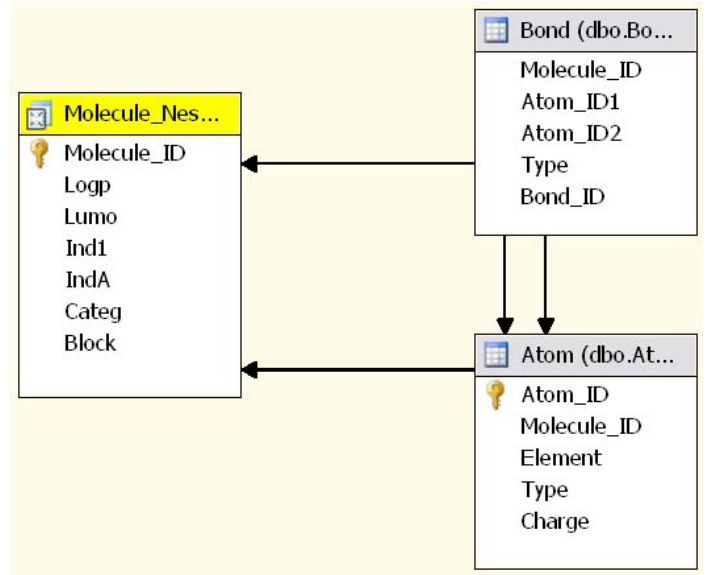

Figure 9: $\quad$ Mutagenesis - DMM schema.

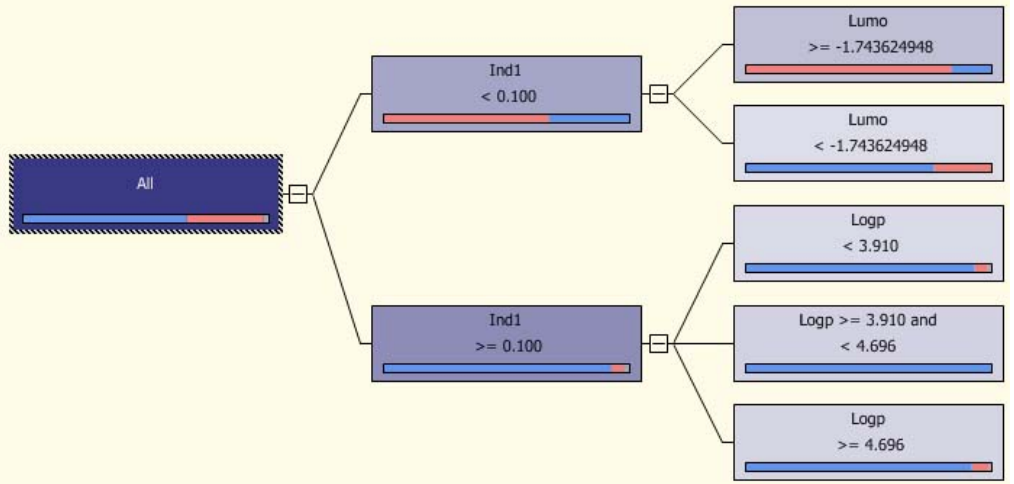

Figure 10: $\quad$ Mutagenesis - MSDT1 Decision Tree. 


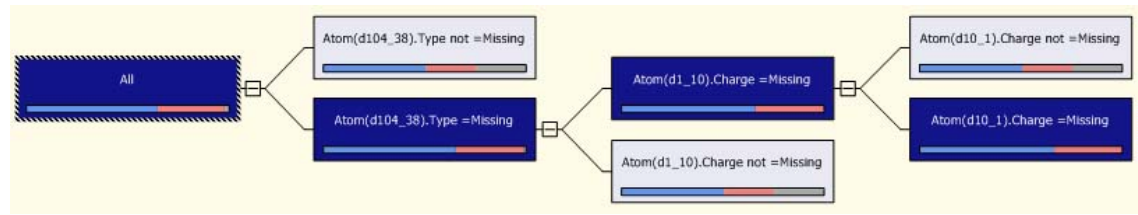

Figure 11: Mutagenesis - MSDT2 Decision Tree.

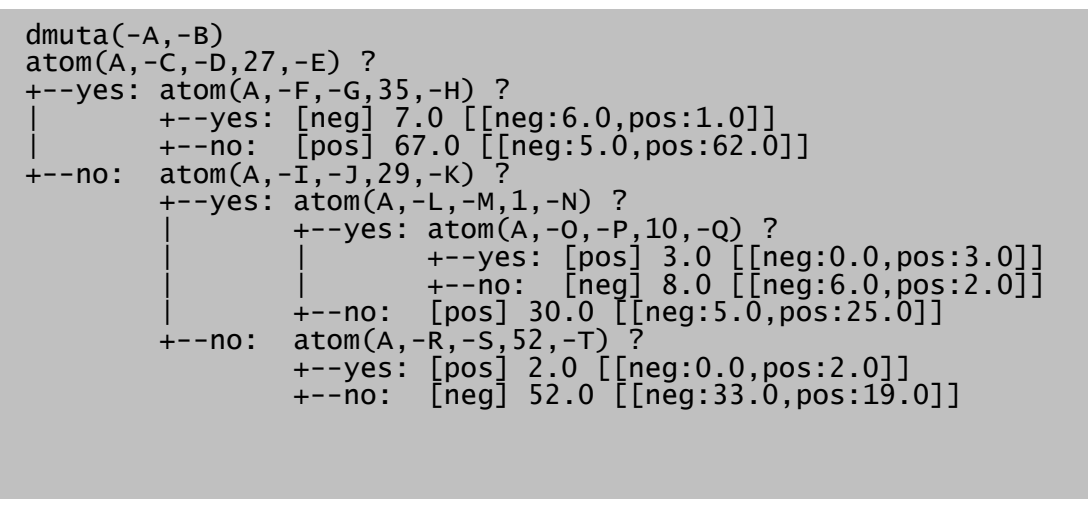

Figure 12: Mutagenesis - TILDE Decision tree.

For this dataset it was produced a sparse matrix with 188 cases and 23,996 attributes. Five of them are the normal attributes, 6,309 for Bond(i).Type and 17,682 for Atom(j).Element, Atom(j).Charge and Atom(j).Type (3*5894). For huge datasets with several nested tables it suggests an explosion on the number of additional attributes that can be unfeasible to handle.

\section{Conclusion and future work}

MSDT showed very good results when using only attributes of the target table, but it seems it cannot handle information in nested tables in a meaningful way: trees thus produced are meaningless and tend to obtain poor predictive accuracy on unseen data. There are, however, a number of questions still unanswered with respect to the plug-in framework of MS SQL and the use of MSDT approach in this framework. In particular, it is unclear what the best approach would be to implement a truly relational algorithm in this framework.

We are running more experiments to better understand the plug-in framework and furthermore we are investigating which algorithm could be the more suitable to be implemented in this framework.

In the experiments we perform, MSDT has equally and even better good predictive accuracy as TILDE, but the trees it gives either ignore the relational information, or use it in a way that yields non-interpretable trees. As such, one could say that its explanatory power is reduced, when compared to a multirelational decision tree learner. 
Finally we conclude that would be useful to integrate multi-relational learners into MSSQL and we are proposing to implement such kind of algorithm to deal with relational DM and to achieve database mining integration.

\section{Acknowledgments}

Claudio L. Curotto is a post-doctoral fellow of the National Council for Scientific and Technological Development (CNPq) and Hendrik Blockeel is a post-doctoral fellow of the Fund for Scientific Research of Flanders, Belgium.

\section{References}

[1] Microsoft Corporation, OLE DB for Data Mining Specification 1.0 Final, $2000 \mathrm{http://www.microsoft.com/downloads/details.aspx?displaylang=en \&}$ familyid=01005 f92-dba1-4fa4-8ba0-af6a19d30217.

[2] Microsoft Corporation, SQL Server Home http://www.microsoft.com/sql/.

[3] Dzeroski, S. \& Lavrac, N. (eds.), Relational Data Mining, Springer, Berlin, Germany, 2001.

[4] Blockeel, H., Top-down induction of first order logical decision trees, $\mathrm{Ph} . \mathrm{D}$. thesis, Department of Computer Science, Katholieke Universiteit Leuven, Leuven, Belgium, 1998.

[5] Leiva, H.A., MRDTL: A Multi-Relational Decision Tree Learning Algorithm, M.Sc. Thesis, Iowa State University, Ames, Iowa, USA, 2002.

[6] Knobbe, A.J., Haas, M. de \& Siebes, A., Propositionalization and Aggregates, in Proc. of the PKDD 2001, Springer, Berlin, Germany, pp 277-288, 2001.

[7] Dzeroski, S. et al., Summer School on Relational Data Mining 2002, http://www-ai.ijs.si/SasoDzeroski/RDMSchool.

[8] Yin, X., Han, J. \& Yang, J., Efficient Multi-Relational Classification by Tuple ID Propagation, in Proc. of the $2^{\text {nd }}$ MRDM Workshop, 2003.

[9] Knobbe, A.J., Siebes, A. \& Van der Wallen, D.M.G., Multi-Relational Decision Tree Induction, in Proc. of the PKDD'99, Springer, Berlin, Germany, pp 378-383, 1999.

[10] Ceci, M., Appice, A. \& Malerba, D., Mr-SBC: a Multi-Relational Naive Bayes Classifier, in PKDD 2003, Lecture Notes in Artificial Intelligence 2838, Lavrac, N., Gamberger, D., Todorovski, L. \& Blockeel, H. (eds.), Springer, Berlin, Germany, pp. 95-106, 2003.

[11] Appice, A., Ceci, M. \& Malerba, D., Mining Model Trees: A MultiRelational Approach, in ILP'03, Lecture Notes in Artificial Intelligence 2835, Horvath, T. and Yamamoto, A. (eds.), Springer, Berlin, Germany, pp 4-21, 2003.

[12] Getoor, L., Learning Statistical Models from Relational Data, Ph.D. Thesis, Stanford University, Stanford, California, USA, 2001.

[13] Muggleton, S.H., Inductive Logic Programming web site - Predicting mutagenicity http://www.doc.ic.ac.uk/ shm/mutagenesis.html. 